Nicolino, J. C., Martz, D. M., \& Curtin, L. (2001). Evaluation of a cognitive-behavioral therapy intervention to improve body image and decrease dieting in college women. Eating Behaviors, 2(4): 353-362. Published by Elsevier (ISSN: 1471-0153).

\title{
Evaluation of a cognitive-behavioral therapy intervention to improve body image and decrease dieting in college women
}

Jill C. Nicolino, Denise M. Martz, and Lisa Curtin

\begin{abstract}
This two-group experimental study evaluated the effectiveness of a cognitive-behavioral body image intervention, adapted from an effective clinical intervention, with normal college females. Participants included nonclinical, freshman college women who were assigned randomly to either the experimental intervention or the control group (brief educational session). Participants were assessed prior to the intervention and again 1 month later on dieting behavior, body image, fear of fat, and anxiety concerning physical appearance. Although it was hypothesized that each of these variables would be lower in the experimental group, none of these results, except for a trend for decreased dieting, were found. Overall these results of slightly reduced dieting behavior are consistent with other research targeting primary and secondary prevention. This intervention's failure to impact body image and eating behaviors of college students illustrates the continuing challenge of eating disorders prevention.
\end{abstract}




\section{INTRODUCTION}

Recently, there have been multiple empirical attempts to improve body image and reduce vulnerability for eating disorders in college students. Such efforts are important because many college students, especially females, appear to have negative body image and some form of disordered eating [Lord, 1994] and [Rockett \& McMinn, 1990]. Once developed, eating disorders may worsen and become lethal, especially if left untreated over time [Neuman \& Halvorson, 1983] and [Shisslak et al., 1990]. Negative body image and resulting dieting behavior are clear precursors to the development of clinical eating disorders [Killen et al., 1993], [Shisslak et al., 1987] and [Thompson, 1996]. Although extreme body dissatisfaction is less common in nonclinical populations, it is still widespread among young women and is not limited to those with eating disorders [Castro \& Goldstein, 1995] and [Rosen et al., 1989]. An average of 60\% of college women diet continuously, and repeated dieting may constitute a risk for both anorexia and bulimia (Killen et al., 1993). Therefore, as dieting and restrained eating continue to be common amongst American women, it is likely there will be even more of an increase in eating pathology [Killen et al., 1993], [Paxton, 1993] and [Sesan, 1989]. Interventions that aim to reduce dieting or improve negative body image, which is related to dieting, are essential to curtail a rise in the prevalence of eating disorders (Rosen et al., 1989).

Treatments for negative body image have proven to be an important adjunctive therapy in the treatment of eating disorders and cognitive-behavioral therapies (CBT) appear to be the most effective. For instance, Rosen, Reiter, and Orosan (1995) found CBT was effective in sustaining improved body image and overall psychological adjustment as compared to a no-treatment control for women with body dysmorphic disorder. Likewise, multiple experimental studies suggest CBT is highly effective in treating severe negative body image and even improves other areas of psychosocial functioning in eating disordered individuals [Grant \& Cash, 1995], [Rosen et al., 1989] and [Thompson, 1996]. Furthermore, several studies have concluded CBT is effective in treating negative body image in overweight or obese women [Robinson \& Bacon, 1996] and [Rosen et al., 1995]. Although these treatments did not address eating, participants reported feeling greater control over eating, feeling less guilty and preoccupied with eating, and binge eating less frequently. Therefore, women can improve negative body image attitudes and behavior with direct intervention without targeting eating, exercise, or weight separately.

Since CBT interventions have been successful in changing negative body image in clinical populations and because negative body image creates a vulnerability for eating disorders, it makes sense to target body image in eating disorders prevention programs. Most preventive interventions involve educational approaches. It is imperative to assess such interventions empirically since despite honorable intentions, they could increase curiosity and worsen the symptoms in some vulnerable individuals (Shisslak et al., 1987) or merely be a waste of time and effort. For example, Paxton (1993) found short-term educational and informational classes were ineffective in reducing disturbed eating, weight loss behaviors, or body dissatisfaction. Similarly, Rosen, et al. (1995) found an educational, nondirective supportive therapy was ineffective in changing body image in normal college women. Likewise, Martz and Bazzini (1999) conducted two controlled studies on the impact of one-shot educational programs for the prevention of eating disorders in college students. Their only consistent effect was a minor 
decrease in dieting behavior for experimental participants compared to controls. Body image was not affected in a consistent manner. Finally, and most importantly, Mann et al. (1997) found an educational preventative intervention for eating disorders, which incorporated both primary and secondary prevention, slightly increased symptoms of eating disorders. They suggest that in trying to reduce the stigma of eating disorders in hopes of encouraging students with problems to seek help, the program may have inadvertently normalized the problem and caused more harm than good.

The purpose of this study was to combine the CBT treatments, proven effective in reducing negative body image in clinical populations, into a brief group preventive intervention for nonclinical college women. Further, this CBT program was compared to a frequently utilized educational program. Success of this program was evaluated by assessing postintervention changes in body image attitudes and dieting-related behavior.

\section{METHOD}

\subsection{Participants}

Neuman and Halvorson (1983) found the peak age of eating disorders onset coincides with the transition from high school to college. Thus, this transitional time period makes a sensible time to target for preventive interventions. Female participants were recruited in return for extra credit from General Psychology courses at a mid-sized Southeastern university (i.e., experimental $\mathrm{n}=45$, control $\mathrm{n}=40$ ). Experimental and control groups were run in five separate sessions consisting of 7 to 10 women each. The mean age was 18.9 (S.D.=2.5). The mean weight was 133.4 (S.D.=23.9) pounds with a range of 95-220 lb. This study was approved by the university IRB and adhered to all ethical guidelines of the American Psychological Association.

\subsection{Materials}

\subsubsection{Demographic questionnaire}

This was a survey created for this study to gather demographic information such as age and race, as well as height, weight, health status, and psychiatric history. In addition, it inquired about friends participating together in the group sessions.

\subsubsection{Body Shape Questionnaire (BSQ)}

The original BSQ is a 34-item body image questionnaire using a 1-6 Likert format with scores ranging from 16 to 96 . It measures the desire to lose weight, body dissatisfaction, and feelings of low self-worth in connection with weight and shape (Cooper, Taylor, Cooper, \& Fairburn, 1986). The coefficients of internal consistency $(\alpha=.97)$, test-retest reliability ( $r=.88)$, and concurrent validity are adequate (Cooper et al., 1986). However, most items on the original 
scale have been found to measure the same underlying construct-concern with shape (Evans \& Dolan, 1993). Therefore, Evans and Dolan (1993) developed an abbreviated 8-item scale with nearly identical psychometrics as the lengthier version. The short form of the BSQ was used in this study to assess concern for body shape.

\subsubsection{Cognitive-Behavioral Dieting Scale (CBDS)}

The CBDS (Martz, Sturgis, \& Gustafson, 1996) is a 14-item, Likert-type scale with scores ranging from 14 to 70 that assesses dieting behaviors and cognitions over the "past 2 weeks." The measure evidences high internal consistency $(\alpha=.95)$ and adequate 2-day test-retest reliability $(r=.92)$ for females. Martz et al. (1996) showed that the CBDS predicts calorie intake and calorie balance above and beyond relevant physical variables, such as body mass index and exercise. It was used in this study to assess calorie-restrictive dieting.

\subsubsection{Physical Appearance State Anxiety Scale (PASTA-state)}

The PASTA-state (Reed, Thompson, Brannick, \& Sacco, 1991) is a 16-item, Likert-type scale with scores ranging from 0 to 64 that assesses state components of anxiety regarding weight (W) and nonweight (NW) relevant body sites (16 sites, $8 \mathrm{~W}$-relevant, $8 \mathrm{NW}$-relevant). Alpha coefficients for factors were uniformly high (W: state=.90; NW: state=.86). The test-retest correlation coefficient for the entire PASTA-state, which also includes a trait scale, was .87 (Reed et al., 1991). Internal consistency ranged from .82 to .92 for the state measure (Thompson, 1996). The state measure was used to assess current level of body image anxiety.

\subsubsection{The Goldfarb Fear of Fat Scale (GFFS)}

The GFFS is a 10-item, Likert-type scale (i.e., 1=very untrue, 4=very true) that is summed for the GFFS score (10=no fear of fat, $40=e x t r e m e$ fear of fat). The GFFS is a quick, reliable scale that is easily administered and scored and has been shown to have discriminant validity, high internal reliability (Cronbach's $\alpha=.85$ ), and high test-retest reliability ( $r=.88$; Goldfarb, Dykens, \& Gerrard, 1985). This measure was used in this study to identify fear of fat related to body image and a tendency towards dieting.

\subsection{DESIGN}

The design was a two-group (experimental vs. control) pretest/posttest design with the following dependent variables: the BSQ (Cooper et al., 1986), the CBDS (Martz et al., 1996), the PASTAstate (Reed et al., 1991), and the GFFS (Goldfarb et al., 1985). Participants were assigned to either experimental or control conditions by randomly alternating sign-up groups at counterbalanced times. 


\subsection{PROCEDURE}

Both experimental and control groups were administered the battery of measures at pretesting. The experimental group, which consisted of five separate groups of 7-10 women each, then participated immediately in a 2-hour group session using CBT techniques described below, implemented by the first author, who was then a master's degree candidate in clinical psychology. To prevent contamination, the groups were asked not to mention the intervention to anyone outside of the group until the study was completed. As a manipulation check, a step-bystep manual of the intervention was used by the presenter and a research assistant to ensure consistent delivery of the intervention for each group. The research assistant observed and coded the presenter's delivery of each intervention component. Furthermore, the research assistant timed the intervention duration, and counted the number of times the group participated in the discussion across all experimental groups to later ascertain if these variables affected the outcome.

The control group, also consisting of five separate groups of 7-10 women, was given a brief educational review on body image and eating disordered behavior to make this group a relevant control. Posttesting for experimental and control groups was conducted 1 month later and participants were awarded extra credit at this time.

\subsubsection{CBT intervention for the experimental group}

The intervention was modeled after the CBT body image therapy of Thompson (1996), but was condensed from a multisession treatment intervention into a 1-hour long preventative intervention:

1. Participants began by completing pretest assessments.

2. The session began by discussing the normal, but unhealthy, prevalence of negative body image in women. Discussion included emotions and beliefs that are involved with negative body image and consequences of behavior that negative body image promotes, such as dieting, checking, and avoidance behaviors.

3. Participants then discussed activators of negative body image, such as sociocultural, peer, and familial factors. They wrote down developmental antecedents of their body image, along with immediate sources of body image distress, if any. Discussion of these antecedents began with the experimenter sharing personal examples relevant to the topic.

4. Participants were then asked to write down and discuss their own appearancepreoccupying rituals. Examples included inspection in a mirror and frequent weighing, as well as time-consuming efforts to manage, repair, or alter one's appearance, usually by perfectionistic grooming regimens. The goal of this discussion was to identify the 
temporary relief these behaviors provide, but emphasize how they perpetuate body image discomfort and distress and, in turn, are actually negative reinforcers.

5. Participants were then asked to write down and discuss what they believed would happen if their distressing body part was openly revealed. The goal of this discussion was to discover the personal maladaptivity of their assumptions, which serve to guide dysfunctional body image thoughts, feelings, and behaviors. The reality of each assumption was challenged and discussed.

6. Participants were then asked to discuss the behaviors they used to compensate for their body image, if any, such as dieting, food restraint, or exercising to lose weight. The goal of this discussion was to improve the client's relationship with her body by expanding her mastery and pleasure in treating her body right and to extinguish disordered eating behaviors. For instance, instead of exercising to lose weight, the pleasurable aspects of exercise were emphasized.

7. Discussion was then geared towards the reduction of distressing self-consciousness. It was explained that these negative thoughts are learned and, therefore, can be unlearned. First, participants were taught relaxation techniques, including deep breathing and progressive muscle relaxation. Next, participants were taught how to refrain from critical self-talk by substituting more objective sensory descriptions of their body parts. The presenter modeled corrective body talk with examples provided by the group, and then discussed its benefits. Furthermore, thought stopping, paired with relaxation, was introduced as a way to extinguish subjective distress. This technique, described by Thompson (1996), refers to (a) stopping the negative self-talk in midstream, (b) looking at activating events and maladaptive private body talk to discover the inherent cognitive errors that are producing body image emotional reactions, and (c) listening to more rational, accurate self-statements that correct the errors.

8. The group ended with discussion of high-risk situations that would cause negative body image relapse, such as interactions with critical friends and relatives, and discussed ways to prevent/cope with such situations. Participants discussed possible situations where relapse may occur, and developed plans to reduce their stress in those situations. The group ended with positive reinforcement and encouragement to incorporate, and share, the techniques discussed to reduce negative body image and disordered eating behaviors.

9. Lastly, information about the Counseling Center's free services for body image, eating disorders, or other psychological needs was distributed.

It was hypothesized that the experimental groups receiving the CBT intervention would show significantly greater reduction in negative body image and dieting behaviors from pretest to posttest as compared to the control group. 


\section{RESULTS}

The intervention was run across several groups of 7-10 participants. Thus, the first analyses were performed on manipulation check variables (i.e., completion of protocol, amount of participant discussion) to assess reliability across all of these presentations and group discussions. To determine if these participation variables affected the dependent variables in the experimental groups, Pearson's correlations were calculated between each variable and the pretest/posttest change score on the CBDS, BSQ, GFFS, and PASTA-state. None of these correlations were significant, suggesting these components of the intervention did not impact results.

Pretest comparisons between groups for body mass index (BMI) were conducted to determine if random assignment yielded equivalent groups for body size. This analysis yielded nonsignificant results $[F(1,83)=0.3, P>.05]$, with a mean of 22.6 across groups, and a range from 16.8 to 37.0. To further ascertain if random assignment yielded equivalent groups, pretest comparisons on the BSQ, CBDS, PASTA-state, and the GFFS were conducted between the experimental and control groups. These comparisons revealed a trend for a higher pretest score for the experimental groups on the BSQ $[F(1,83)=3.1, P<.08]$ with no significant differences found on the PASTA-state, the CBDS, and the GFFS (all $P^{\prime} \mathrm{S}>.05$ ).

Although random assignment was used in this study, a covariance analysis was used to reduce any group variability discovered at pretest (Stevens, 1992). A series of analyses of covariance (ANCOVA) were used to assess group differences between the experimental group, which received the CBT intervention, and control group, that received the educational review, on each of the following posttest variables with corresponding pretest used as the covariate: BSQ $[F(1,81)=2.0, P>.05]$, CBDS $[F(1,81)=3.3, P<.08]$, PASTA-state $[F(1,81)=0.58, P>.05]$, and GFFS $[F(1,81)=1.2, P>.05]$. In addition, ANCOVAs controlling for BMI as a covariate were conducted on posttest differences, but this did not significantly change the outcome. Pretest and posttest means and standard deviations are presented in Table 1. Fig. 1 illustrates the trend for lower CBDS scores in the experimental group versus controls. 
Table 1. Means and standard deviations of pretest- and posttest-dependent variables in experimental vs. control groupsValues in parentheses represent standard deviations.

\begin{tabular}{|c|c|c|c|c|}
\hline \multirow{2}{*}{ Dependent val } & \multicolumn{2}{|c|}{ Experimentals $(n=40)$} & \multicolumn{2}{|c|}{ Controls $(n=45)$} \\
\hline & Pretest & Posttest & Pretest & Posttest \\
\hline BSQ & $48.8(2.2)$ & $42.8(1.9)$ & $42.9(2.6)$ & $40.5(2.5)$ \\
\hline CBDS & $38.9(1.7)$ & $34.8(1.9)$ & $35.0(1.7)$ & $34.2(2.0)$ \\
\hline PASTA-state & $21.7(1.7)$ & $20.8(1.5)$ & $19.3(2.1)$ & 18.5 (1.8) \\
\hline GFFS & $20.2(1.0)$ & $19.6(1.0)$ & $18.9(0.9)$ & $17.7(1.0)$ \\
\hline
\end{tabular}

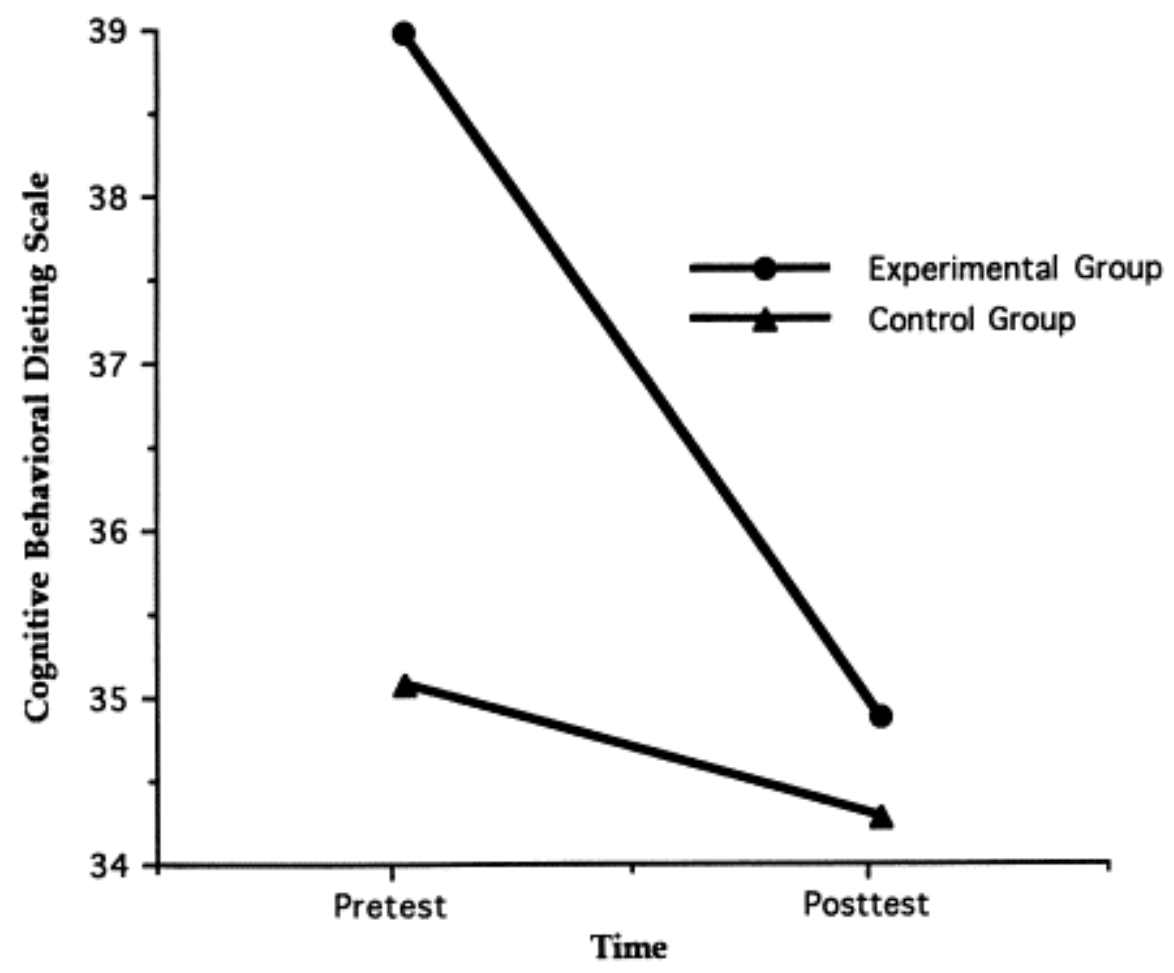

Fig. 1. Group differences on the CBDS over time. 


\section{DISCUSSION}

The CBT intervention targeted prevention of eating disordered behavior in college women by attempting to reduce dieting behaviors and negative body image, both of which are key factors in the development and the preservation of clinical eating disorders [Lord, 1994], [Neuman \& Halvorson, 1983], [Sesan, 1989] and [Shisslak et al., 1987]. This research adapted an intervention found to be effective in treating clinical body image disorders and applied it to the prevention of such disorders in college females. Results revealed there was no significant change for body image anxiety, body shape concerns, or fear of fat, although a trend was found in the expected direction for dieting. These nonsignificant results are consistent with past studies of preventive interventions, but may also be a function of methodological limitations.

Due to the use of a nonclinical population, extremely elevated pretest scores were not expected and scores on the dependent measures may have had little opportunity to change given a possible floor effect on the dependent measures. Consequently, the impact of the intervention may not have been adequately measured by the dependent variables. The BSQ, the GFFS, and the PASTA-state all measure inherent components of body image anxiety, which may be much more longstanding than behavioral components of dieting across the past 2 weeks. Therefore, the trend found on the CBDS may be attributed to the fact that it was created to be a sensitive measure of behavioral change (Martz et al., 1996). Within this intervention, there was a strong emphasis on the negative aspects of dieting, such as the lowering of metabolism and weight gain following each dieting attempt. Education about dieting consequences, therefore, may be more influential on behavioral changes than on long-standing or intrinsic changes in body image. Similar results have been found in other prevention studies in which negative body image did not improve, despite a decrease in dieting behavior (Martz \& Bazzini, 1999).

In addition, the intervention used in this study was condensed from Thompson's (1996) multisession treatment paradigm into a very brief preventative model. Consequently, the preventative model may have been too abbreviated to make any significant impact. Long-term follow-ups are needed to assess for any possible body image change over time or further decrease in dieting. The 1-month follow-up in the present study may have not allotted enough time to show any impact, or any impact may have decayed across the month.

Although results proved nonsignificant, this research contributed to eating disorder prevention research on several accounts. Although negative body image may be a more difficult component to change than dieting behavior (Martz \& Bazzini, 1999), self-perception theory states that self-concept may be impacted by behavior (Bem, 1972). Therefore, personal changes in behavior, such as reduced dieting, may be very important precursors in the process of changing negative body image and self-concept.

This research was also one of the first to test a cognitive-behavioral-emotional style of prevention that aimed at group and peer interaction, as Griffiths and Farnhill (1996) suggest. Modification of weight, dieting attitudes, and practices can be considered very important in the primary prevention of dieting disorders (Griffiths \& Farnhill, 1996). Therefore, the trend for reduced dieting, which has been found in several prevention studies, could with the employment 
of longer term follow-up assessments be shown to reduce vulnerability for anorexia and bulimia [Killen et al., 1993], [Paxton, 1993] and [Sesan, 1989].

Consistent with previous research targeting primary or secondary prevention of eating disorders, this study failed to find any meaningful impact. Unlike other research, however, this intervention directly borrowed (albeit in a condensed format) from Thompson's (1996) successful treatment program for clinically severe body image disorders. It is possible that the strategies that are effective in treating severe negative body image are too diluted in a condensed format or do not fit milder body image concerns. Likewise, the previously mentioned problem of range restriction may be operating when using such interventions with nonclinical individuals. Nonetheless, the use of preventive intervention groups aimed at peer discussion and supportive interactions including CBT strategies has not been adequately tested. Future research should focus on these important elements and their possible effectiveness for primary and secondary prevention through reduced dieting behaviors by using frequent assessments across time including long-term follow-up studies. 


\section{REFERENCES}

Bem, D. J. (1972). Self perception theory. In: L. Berkowitz (Ed.), Advances in experimental social psychology, (vol. 6, pp. 1-62). San Diego, CA: Academic Press.

Castro, J. M., \& Goldstein, S. (1995). Eating attitudes and behaviors of pre- and postpubertal females: clues to the etiology of eating disorders. Physiology \& Behavior, 58, 15-23.

Cooper, P. J., Taylor, M. J., Cooper, Z., \& Fairburn, C. G. (1986). The development and validation of the body shape questionnaire. International Journal of Eating Disorders, 6, 485494.

Evans, C., \& Dolan, B. (1993). Body shape questionnaire: derivation of shortened "alternate forms." International Journal of Eating Disorders, 13, 315-321.

Goldfarb, L. A., Dykens, E. M., \& Gerrard, M. (1985). The Goldfarb Fear of Fat Scale. Journal of Personality Assessment, 49, 329-332.

Grant, J., \& Cash, T. F. (1995). Cognitive-behavioral body image therapy: comparative efficacy of group and modest-contact treatments. Behavior Therapy, 26, 69-84.

Griffiths, R. A., \& Farnhill, D. (1996). Primary prevention of dieting disorders: an update. Journal of Family Studies, 2, 179-191.

Killen, J. D., Taylor, C. B., Hammer, L. D., Litt, I., Wilson, D. M., Rich, T., Hayward, C., Simmonds, B.,

Kraemer, H., \& Varady, A. (1993). An attempt to modify unhealthful eating attitudes and weight regulation practices of young adolescent girls. International Journal of Eating Disorders, 13, 369-384.

Lord, M. G. (1994). Forever Barbie. The unauthorized biography of a real doll. New York: Avon Books.

Mann, T., Nolen-Hoeksema, S., Huang, K., Wright, A., Hanson, K., \& Burgard, D. (1997). Are two interventions worse than none? Joint primary and secondary prevention of eating disorders in college females. Health Psychology, 16, 215-225.

Martz, D. M., \& Bazzini, D. G. (1999). Eating disorder prevention programming may be failing: evaluation of two, one-shot programs. Journal of College Student Development, 40 (1), 32-42.

Martz, D. M., Sturgis, E. T., \& Gustafson, S. B. (1996). Development and preliminary validation of the Cognitive Behavioral Dieting Scale. International Journal of Eating Disorders, 19, 297309.

Neuman, P. A., \& Halvorson, P. A. (1983). Anorexia nervosa and bulimia. A handbook for counselors and therapists. New York: Van Nostrand Reinhold. 
Paxton, S. J. (1993). A prevention program for disturbed eating and body dissatisfaction in adolescent girls: a 1 year follow-up. Health Education Research Theory \& Practice, 8, 43-51.

Reed, D., Thompson, J. K., Brannick, M. T., \& Sacco, W. P. (1991). Development and validation of the Physical Appearance State and Trait Anxiety Scale (PASTAS). Journal of Anxiety Disorders, 5, 323-332.

Robinson, B. E., \& Bacon, J. G. (1996). The "If only I were thin. . ." treatment program: decreasing the stigmatizing effects of fatness. Professional Psychology: Research and Practice, 27, 175-183.

Rockett, G., \& McMinn, K. (1990). You can never be too rich or too thin: how advertising influences body image. Journal of College Student Development, 31, 278.

Rosen, J. C., Orosan, P., \& Reiter, J. (1995). Cognitive behavior therapy for negative body image in obese women. Behavior Therapy, 26, 25-42.

Rosen, J. C., Reiter, J., \& Orosan, P. (1995). Cognitive-behavioral body image therapy for body dysmorphic disorder. Journal of Consulting and Clinical Psychology, 63 (2), 263-269.

Rosen, J. C., Saltzberg, E., \& Srebnik, D. (1989). Cognitive behavior therapy for negative body image. Behavior Therapy, 20, 393-404.

Sesan, R. (1989). The bulimic college student: evaluation, treatment, and prevention. New York: Haworth Press.

Shisslak, C. M., Crago, M., \& Neal, M. E. (1990). Prevention of eating disorders among adolescents. American Journal of Health Promotion, 5, 100-106.

Shisslak, C. M., Crago, M., Neal, M. E., \& Swain, B. (1987). Primary prevention of eating disorders. Journal of Consulting and Clinical Psychology, 55, 660-667.

Stevens, J. (1992). Applied multivariate statistics for the social sciences. Hillsdale, NJ: Lawrence Erlbaum Associates.

Thompson, J. K. (1996). Body image, eating disorders, and obesity. Washington, DC: American Psychological Association. 